Conference Paper

\title{
Analysis and experiential verification of power loss in joint area of laminated transformer core
}

Kalinin, Y., Chivenkov, A., Vagapov, Y., and Anuchin, A.

This is a paper presented at the 56th IEEE Int. Universities Power Engineering Conference UPEC2021, Middlesbrough, UK, 31 Aug. - 3 Sept. 2021

Copyright of the author(s). Reproduced here with their permission and the permission of the conference organisers.

\section{Recommended citation:}

Kalinin, Y., Chivenkov, A., Vagapov, Y., and Anuchin, A. (2021), 'Analysis and experiential verification of power loss in joint area of laminated transformer core.' In: Proc. 56th IEEE Int. Universities Power Engineering Conference UPEC-2021, Middlesbrough, UK, 31 Aug. - 3 Sept. 2021, pp. 1-6, doi: 10.1109/UPEC50034.2021.9548245 


\section{Analysis and Experiential Verification of Power Loss in Joint Area of Laminated Transformer Core}

\author{
Yevgeniy Kalinin \\ Institute of Electrical Power Engineering \\ Nizhny Novgorod State Technical University \\ Nizhny Novgorod, Russia \\ Yuriy Vagapov \\ Faculty of Art, Science and Technology \\ Glyndwr University \\ Wrexham, UK
}

\author{
Alexandr Chivenkov \\ Institute of Electrical Power Engineering \\ Nizhny Novgorod State Technical University \\ Nizhny Novgorod, Russia \\ Alecksey Anuchin \\ Department of Electric Drives \\ Moscow Power Engineering Institute \\ Moscow, Russia
}

\begin{abstract}
This paper discusses the processes of power loss development in the joint area of the laminated transformer core due to eddy currents produced by the normal magnetic flux. Normal magnetic flux is directed in perpendicular to the plane of the laminations and the dominating factor in the power loss formation in the joint area. The analytical approach was verified by a set of tests where two experimental setups have been employed to investigate power loss in the laminations under various conditions. The tests provided data on the power loss produced by the normal flux in relation to the lamination width and lamination overlap length. It was shown that the power loss in the joint area significantly depends on the lamination width and is independent of lamination overlap.
\end{abstract}

Keywords-laminated core; loss in joint area; eddy current loss; severe skin effect; normal magnetic flux

\section{INTRODUCTION}

Electromagnetic loss often referred to as no-load loss, is an important characteristic of a power transformer representing a constant component of energy losses. A significant improvement in the transformer efficiency performance is achieved due to the implementation of modern textured cold-rolled electrical steels ensuring low values of specific magnetic losses and an increased field density in the rolling direction. New types of electric steel enable to enhance the transformer properties include advanced material such as Hi-B, ZDKH, magnetoactive coating, laser scribed steel, amorphous steels [1]-[3]. Mitre cut joint technology introduced in the magnetic core design provided a further reduction of power loss in the transformer joint area [1]. However, the power transformers are still responsible for approx. $1 / 3$ of power loss occurred in the transmission and distribution stages of the grid [4]. It demands further improvement in magnetic core design focused to reduce the transformer loss rate and, therefore, increase the efficiency of the grid operation.

Textured electrical steels are characterised by cube-onedge texture, pronounced anisotropy of magnetic properties, and stripe domain structure [2],[5]. The magnetic properties of the textured steels are sensitive to mechanical stress and speed of magnetisation reversal $d B / d t$ [5]. The joints in laminations degrade the characteristics of the magnetic core in comparison with the steel properties. Although considerable efforts have been made to improve the joint designs, the structure of the power loss increase in the joint area of the laminated magnetic cores is poorly studied. Further progress in the core joint design requires a better understanding of magnetic field performance and the power loss formation in the joint area [6].
Investigation of additional losses in the joints of laminated magnetic core presented in [7]-[15] is based on conventional, widely recognised structure of the power loss in electrical steels proposed by Bertotti [2],[16]. However, this approach does not provide detailed analysis of the losses associated with the magnetic flux transfer through adjacent layers (from lamination to lamination) of the core.

A new approach suggested in [6] represents the power loss occurred in the joint area as an additional loss $\Delta P$ gained in comparison to the same jointless (solid) core. This increase $\Delta P$ consists of two components: (1) the increase in the combined hysteresis-eddy current losses $\Delta P_{H E \tau}$ related to the compression of the tangential (main) magnetic flux $\Phi_{\tau}$ in the joint area, and (2) power loss due to eddy currents $P_{E n}$ produced by the normal flux $\Phi_{n}$ directed in perpendicular to the plane of the laminations. The separation of the power losses was obtained from a series of experiments where the power loss in a circle laminated magnetic core made by isotopic electrical steel was investigated under two test conditions. Initially, the core was tested without joints to obtain the reference value of the power loss $P$; then, the laminations were cut into four sections and assembled with joint overlapping to determine the increase in the power loss $\Delta P$. The tests of the cut magnetic core were conducted for three different numbers of laminations per step $m=1,2,8$.

Fig. 1a [6] demonstrates curves of the relative increase (in p.u.) of the power loss in the joint area $\delta P=\delta P_{H E \tau}+\delta P_{E n}$ vs. magnitude of the magnetic field density $B_{M}$ obtained from investigation of the magnetic core made of isotopic electrical steel $2412(3 \% \mathrm{Si}$; specific loss is $4.2 \mathrm{~W} / \mathrm{kg}$ at $B_{M}=1.5 \mathrm{~T}$ and $f=50 \mathrm{~Hz}$ ), where the core lamination width is $b=60 \mathrm{~mm}$ and the number of laminations per step $m=8$. It can be seen that the character of the loss components are different: the component $\delta P_{H E \tau}\left(B_{M}\right)$ is reduced at higher values of the magnetic field density; the component $\delta P_{E n}\left(B_{M}\right)$ has the maximum value at $B_{M}=1.0-1.1 \mathrm{~T}$ (approx.) which is corresponding to the knee area of the magnetising curve. [6] also reports that the reduction in the lamination width $b$ decreases the power loss related to the normal flux as $\delta P_{E n}\left(B_{M}\right) \propto b^{0.5}$. The character of relative power loss cures $\delta P\left(B_{M}\right)$ for the smaller numbers of $m$ $(m=1 ; 2)$ is the same as shown in Fig. 1a. It suggests that the component of the relative increase in power loss $\delta P_{H E \tau}$ is proportional to the level of specific loss of the electrical steel, whereas the component $\delta P_{E n}$ depends on the specific electrical conductivity $\gamma$, which is the same for steels having $3 \%$ of Si. The specific electrical conductivity is independent of the core geometry including lamination width $b$.

Fig. $1 \mathrm{~b}[6]$ shows the curves of the relative increase in power losses obtained for transformer steel 3406 (specific 


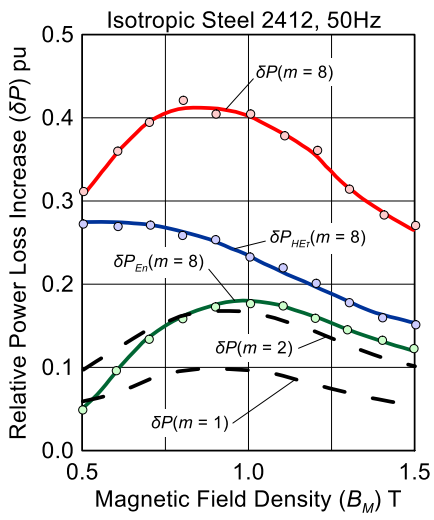

(a)

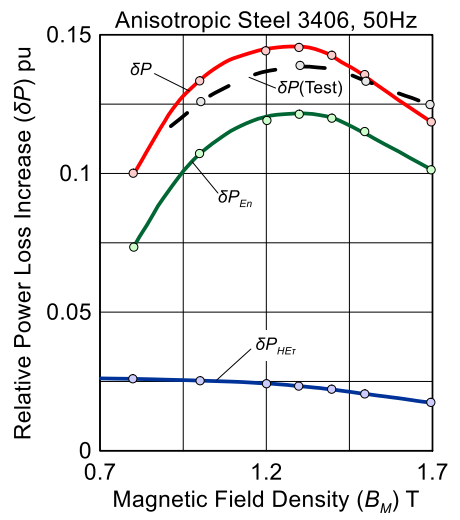

(b)

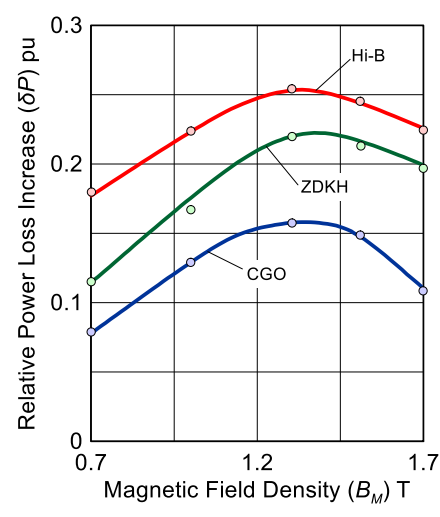

(c)

Fig. 1. Relative power loss increase $\delta P$ vs. magnetic field density $B_{M}$ at $50 \mathrm{~Hz}$ for (a) Isotropic Si steel 2412; (b) Anisotropic Si steel 3406; (c) high permeability Si steel (HiB), laser scribed high permeability Si steel (ZDKH), conventional grain-oriented Si steel (CGO). Data from [6],[7].

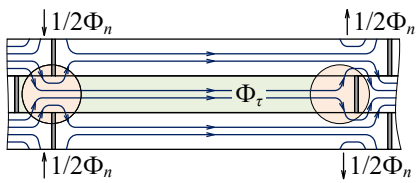

(a)

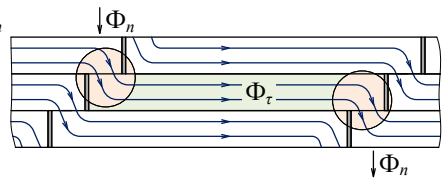

(b)

Fig. 2. Normal flux $\Phi_{n}$ components at the ends of the laminations in (a) "butt-lap" and (b) "step-lap" magnetic core assemblies.

power loss is $1.25 \mathrm{~W} / \mathrm{kg}$ at $B_{M}=1.7 \mathrm{~T}$ and $f=50 \mathrm{~Hz}$ ). It can be seen that the component related to the normal flux $\delta P_{E n}$ dominates in the total losses in the joints having the maximum value at $B_{M}=1.3-1.4 \mathrm{~T}$ [6].

An improvement in the specific loss of modern transformer steels leads to a further relative increase of power loss component $\delta P_{E n}$ in the joints. [7] provides an experimental comparison of losses in magnetic cores made of textured steels with different levels of specific losses. Three identical three-phase laminated magnetic cores (lamination width is $b=100 \mathrm{~mm}$ ) made of different electrical steels were investigated: (1) conventional grainoriented $\mathrm{Si}$ steel (CGO); (2) high permeability $\mathrm{Si}$ steel (HiB), and (3) laser scribed high permeability $\mathrm{Si}$ steel $(\mathrm{ZDKH})$. Specific loss in the rolling direction for $\mathrm{CGO}$ is $1.21 \mathrm{~W} / \mathrm{kg}$, $\mathrm{HiB}-1.01 \mathrm{~W} / \mathrm{kg}$, and ZDKH $-0.95 \mathrm{~W} / \mathrm{kg}$. It was measured at a magnetic field density of $B_{M}=1.7 \mathrm{~T}$ and a frequency of $f=50 \mathrm{~Hz}$. The report [7] confirms that the steels having lower specific losses in the rolling direction demonstrate the larger values of the relative increase in specific power loss $\delta P$ in the joints. Fig. 1c shows the relative increase in specific power loss for these electrical steels. It can be seen that the character of the curves in Fig. 1c is similar to the curves shown in Fig. 1a and Fig. 1b. One of the reasons for such similarity is the share increase of the component $\delta P_{E n}$ due to improvement in the specific loss in the rolling direction.

It is observed that the normal flux $\Phi$ n brings a significant impact on the increase in power loss in the joint area of the "step-lap" mitre cut transformer cores. [18] experimentally proved that the losses in the magnetic core (lamination width $b=165 \mathrm{~mm}$ ) of the three-phase power transformer (300 KVA) made of electrical steel $27 \mathrm{M} 4$ is higher by $15 \%$ for "step-lap" joints comparing to "butt-lap" core joints. This study assumed that the eddy current losses produced by a normal flux depend on $\left(t_{j}\right)^{2}$, where $t_{j}$ is the joint overlap length.

A variety of studies [10]-[13] providing numerical analysis of electromagnetic field and the power loss in the core joints formulate the problem using the joint overlap

length $t_{j}$ instead of the lamination width $b$. 3-D modelling was applied for numerical analysis of losses in the transformer joint area [14],[15]. However, the influence of the lamination width $b$ was not taken into account in these studies.

\section{Analysis of Power Loss Produced by Normal Flux}

In terms of performance of the normal flux $\Phi_{n}$ in the joint area, all laminations of the core are practically in the same conditions. Equal normal fluxes $\Phi_{n}$ are concentrated in the overlapping areas of adjacent sheets at the ends of each lamination. Fig. 2 shows the normal flux distribution in the lamination joints assembled using "butt-lap" and "steplap" methods [18]. The arrows indicate the direction of the flux in the laminations at some arbitrary moment in time; in this illustration, the flux through the air-gaps are considered negligible. The thickness of the step in the laminated core is determined as $m \times d$, where $m$ is the number of laminations in the step, $d$ is the thickness of lamination. The circles in Fig. 2 highlight the areas where the normal flux passes through the adjacent layers (from lamination to lamination) of the magnetic core. It shows that the distribution of the normal flux in the overlapping area is significantly different for "butt-lap" and "step-lap" assemblies.

When the laminations are "butt-lapped" (Fig. 2a), the tangential magnetic flux $\Phi_{\tau}$ in the joint area is compressed and divided onto two equal (assuming the symmetry in the joint area) normal fluxes $1 / 2 \Phi_{n}$. Each half of the normal flux passes into adjacent layers (up and down) at the ends of the laminations. The upper half fluxes (as well as the lower half fluxes) at both ends of the lamination have opposite directions.

When the laminations are "step-lapped" (Fig. 2b), the tangential magnetic flux $\Phi_{\tau}$ in the joint area is not compressed and turns in one direction passing into adjacent layers at the full value of $\Phi_{n}$ [9]. Under equal operational circumstances, the normal flux in the "step-lapped" core is in two times bigger in comparison to the "butt-lap" design and has the same direction at both ends of the lamination.

Magnetic flux $\Phi$ of an individual transformer limb (or yoke) consisting of laminations can be determined (at any moment of time) as follows

$$
\Phi=\sum \Phi_{\tau}=\sum \Phi_{n}+\sum \Phi_{\delta}
$$

where $\Phi_{\tau}, \Phi_{n}, \Phi_{\delta}$ are the components of the flux in a lamination $\left(\Phi_{\tau}\right.$ is the tangential flux; $\Phi_{n}$ is the normal flux; $\Phi_{\delta}$ is the flux through the air-gap of the joint). Thus, the 
total value of the magnetic flux $\Phi_{n}$ creating eddy current losses in the joint area $P_{E n}$ is comparable to the magnitude of the flux $\Phi\left(\sum \Phi_{n} \leq \Phi\right)$. In this case, the magnitude of the cross-section average field density produced by the normal flux $B_{M n}$ is tens times less than the field density $B_{M}$ in the core. The smaller value of the field density is due to a significantly larger cross-section of the joint overlapping area $S_{n}$ in comparison to the cross-section of the lamination step $S_{\tau}$. The ratio $S_{n} / S_{\tau}=t_{j} /(m \times d)>>10$ as shown in Fig. 3 .

The losses in the joint area $\Delta P_{H E \tau}$ and $P_{E n}$ occur in the orthogonal planes of the electric steel core. The steel medium has linear electrical conductivity (it is assumed that the specific electrical conductivity of the steel $\gamma=$ const) at low values of magnetic field density $B_{M n}$ and, therefore, these processes can be studied using a superposition method. Hence, the analysis of losses in joints $P_{E n}$ is considered as an independent problem. This approach is relevant due to the high level of power losses $P_{E n}$ in the joints of the laminated core made of textured steel [6].

The linear dimension affecting the eddy-current producing due to the normal flux $\Phi_{n}$ is the width of the core plates $b[6]$, but not the length of the lamination overlap $t_{j}$ as suggested in [1], [9]-[13]. In fact, $t_{j}$ determines only the width of the area where the magnetic flux $\Phi_{n}$ flows into the adjacent layers but does not limit the eddy currents produced by the flux $\Phi_{n}$ (Fig. 3). Dynamic magnetisation reversal processes occur at low values of $B_{M n}$ corresponding to an insignificant nonlinearity of magnetic permeability $\mu$.

The parameter called dumping factor $\xi$ is introduced in [17] to analyse the skin effect produced by a plane electromagnetic wave freely penetrating into an infinite long conducting sheet having $\mu=$ const.

$$
\xi=l \sqrt{\pi f \mu \gamma}
$$

where $l$ is the smallest transverse dimension of lamination; $f$ is the frequency; $\mu$ is the magnetic permeability of material $\mu=\mu_{r} \times \mu_{0} \quad\left(\mu_{r}\right.$ is relative permeability; $\mu_{0}$ is the magnetic constant $\left.4 \pi \times 10^{-7} \mathrm{H} / \mathrm{m}\right) ; \gamma$ is the specific electrical conductivity $\gamma \approx 2 \times 10^{6} \mathrm{~S} / \mathrm{m}$ for $3 \%$ Si steels.

For the tangential magnetic flux $\Phi_{\tau}$ in the laminated magnetic core, the smaller transverse dimension $l$ shown in (2) is the thickness of the lamination $d$. The process of magnetization reversal of electrical steels along the rolling direction at an industrial frequency of $50 \mathrm{~Hz}$ is characterised by the dumping factor $\xi<2$. Specific eddy current losses $P_{E \tau}$ produced by the tangential flux $\Phi_{\tau}$ are determined by the classical formula [2],[5],[6],[16],[17]:

$$
P_{E \tau}=\frac{\pi^{2} f^{2} \gamma}{6 \sigma} B_{M}^{2} d^{2} \propto B_{M}^{2} d^{2}
$$

where $\sigma$ is the material mass density; $d$ is the lamination thickness (the smallest transverse dimension for the tangential magnetic flux).

For the normal flux $\Phi_{n}$ flowing in perpendicular direction to the lamination plane, the smallest transverse dimension is the width of the lamination $b$. It is quite difficult to determine experimentally the magnetic permeability $\mu_{\perp}=\mu_{\perp r} \times \mu_{0}$ of the electrical steel in the direction of the normal flux $\Phi_{n}$. According to the results reported in [19]-[21], the relative permeability $\mu_{\perp r}$ has a value in the range between 30 to 170 . The dynamics process of magnetisation reversal occurs under the condition of a severe skin effect at the damping factor $\xi>4$. If the lamination geometry is assumed as "infinite plate" [17],

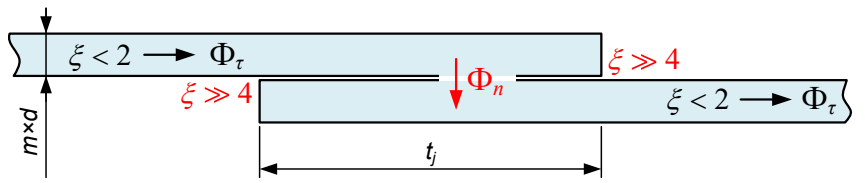

Fig. 3. Transition of the tangential flux $\Phi_{\tau}$ into the normal flux $\Phi_{n}$.

then the specific losses due to eddy currents $P_{E n}$ produced by the flux $\Phi_{n}$ under the severe skin effect condition is expressed as follows:

$$
P_{E n}=\frac{\pi^{1.5} f^{1.5}}{2 \sigma} \sqrt{\frac{\gamma}{\mu_{\perp}}} B_{M n}^{2} b \propto B_{M n}^{2} b
$$

Thus, a significant part of losses in the joint area is produced under severe skin effect conditions. In the laminations overlap area, the value of the dumping factor is quite high $\xi>>4$. It was experimentally found [6] that the power loss $P_{E n}$ is proportional to the width of the lamination as $b^{0.5}\left(P_{E n} \propto b^{0.5}\right)$ which is contradicting to (4). The performance of the normal flux $\Phi_{n}$ in the joint area (Fig. 3) is fundamentally different to the flux obtained from the skin effect problem for a conventional conducting plate [17]:

- the flux is concentrated in the narrow overlap area at the ends of the lamination;

- the transition of the flux from lamination to lamination $\Phi_{\tau} \rightarrow \Phi_{n} \rightarrow \Phi_{\tau}$ occurs under a sharp change in the dumping factor: $(\xi<2) \rightarrow(\xi>>4) \rightarrow(\xi<2)$ as shown in Fig. 3.

\section{EXPERIMENTAL SETUPS}

In order to verify the proposed analytical approach, the power loss development $\mathrm{P}_{E n}$ was experimentally investigated to understand its relation to the lamination dimensions and overlap area. Two experimental setups were designed and built to provide testing of the lamination samples under application of the normal flux through two opposite sides of a rectangular electrical steel sheet as shown in Fig. 4 and Fig 5. Both setups produce the ac fluxes through each side of the sample directed perpendicular to the lamination plane, equal in magnitude and performed under the severe skin effect condition. The advantage of the experimental setups is the possibility to analyse power loss $P_{E n}$ in the joint area produced by the normal flux $\Phi_{n}$ solely and to exclude the influence of other factors. A similar experimental approach was used in [22] to study eddy current losses $P_{E n}$ generated by the normal leakage flux in the magnetic core of a power transformer.

Setup 1 consists of a circle magnetic core cut into two C-shape yokes (Fig. 4). The cross-sectional area of the core is $100 \times 16 \mathrm{~mm}$. The magnetising windings have 660 turns each. The magnetic circuit of setup 1 is symmetrical that provides equal normal fluxes $\Phi_{n}$ through the lamination sample and eliminates tangential flux component $\Phi_{\tau}$. The fluxes through each end of the sample are arranged in the opposite directions. The length of the sample applicable for this setup is $L=165 \mathrm{~mm}$, the width of the setup yoke is $L_{Y}=16 \mathrm{~mm}$.

Setup 2 consists of two identical U-shaped magnetic cores (Fig. 5). The cross-section area of the yoke producing the normal flux through the sample is $100 \times 26 \mathrm{~mm}$. The exciting windings (240 turns each) are connected in parallel to a $50 \mathrm{~Hz}$ ac voltage source to generate two equal ac magnetic fluxes in each magnetic core. Fig. 6 shows the electrical circuit diagram of the experimental setup. The direction of the fluxes in the magnetic cores depends on the 


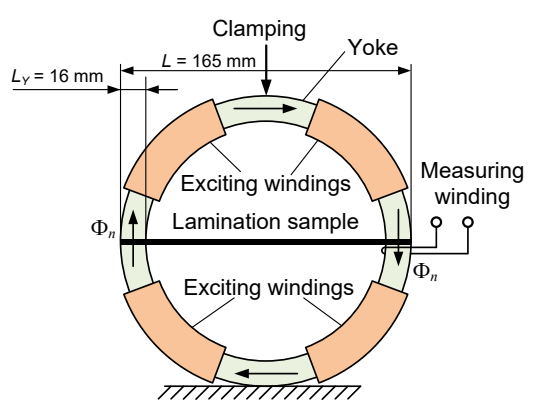

Fig. 4. Experimental setup 1 (opposite flux direction arrangement).

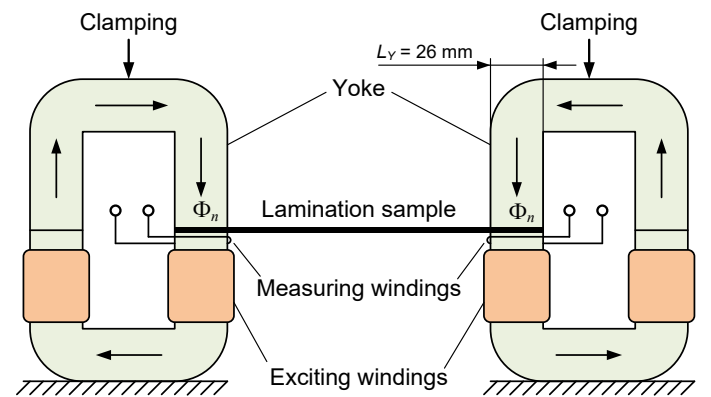

Fig. 5. Experimental setup 2 (the same flux direction arrangement).

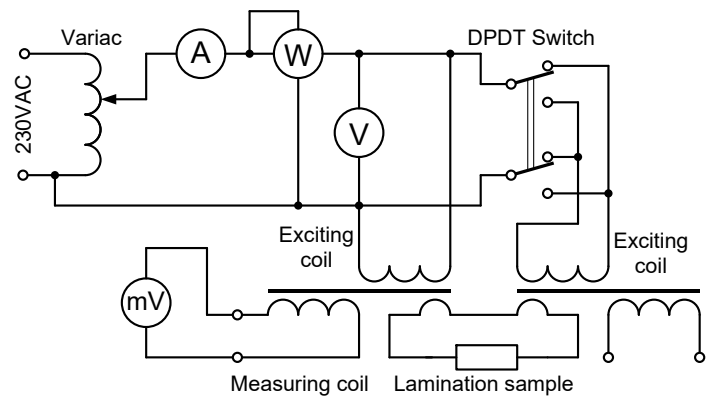

Fig. 6. Electrical circuit of the experimental setup 2.

position of the switch (DPDT switch in Fig. 6) and it can be arranged in the same or opposite directions. Although setup 2 was designed to investigate the samples under two different arrangements of the flux direction, in the present study it has been used to test the laminations at the same direction of the fluxes only. The length of the sample applicable for this setup can be varied, the width of the setup yoke is $L_{Y}=26 \mathrm{~mm}$.

Experimental setup 1 is equipped with a measuring winding ( 3 turns) installed on one setup yoke to monitor the flux amplitude. A low power factor wattmeter D522 was used to acquire the test readings. The sample material is a conventional transformer steel grade 3406 . It is a $3 \% \mathrm{Si}$ electrical steel having the specific electrical conductivity $\gamma \approx 2 \times 10^{6} \mathrm{~S} / \mathrm{m}$ and the relative permeability $\mu_{\perp r}=150$. The size of the sample is $165 \times 100 \mathrm{~mm}$; the sample thickness is $d=0.35 \mathrm{~mm}$. The properties of the sample ensure the setup operation under the dumping factor $\xi>>4(\xi=24)$.

In order to conduct the tests, the ends of the lamination sample were fixed between the yokes of C-shaped (Fig. 4) or U-shaped cores (Fig. 5). The lower yokes of the setups are attached to the base, the upper yokes are movable and fixed using the clamps. To avoid the influence of the temperature variation on sample electrical conductivity, all measurements were conducted under the condition $\mathrm{T}^{\circ} \mathrm{C}=$ const. Fig. 7 shows a photo of the experimental setups and the steel rectangular lamination samples.

The power balance for the experimental setups is expressed as follows:

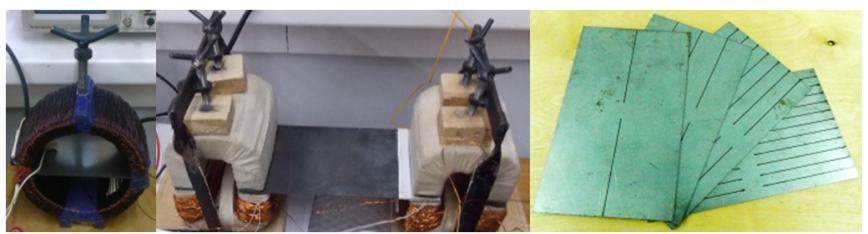

Fig. 7. Experimental setups and the steel rectangular lamination samples.

$$
P=P_{C}+P_{S}+P_{E n}
$$

where $P$ is measured active power [W]; $P_{C}=I^{2} \times\left(R_{M}+R_{W}\right)$ is loss in copper; $I$ is rms value of the current in the magnetising windings $[\mathrm{A}] ; R_{M}$ is resistance of the magnetising windings $[\Omega] ; R_{W}$ is resistance of the current coil of the low power factor wattmeter $[\Omega] ; P_{S}$ is loss in steel (magnetic core) $[\mathrm{W}] ; P_{E n}$ is eddy current loss in the lamination sample due to the normal flux [W].

The power loss in magnetic core $P_{S}$ is determined from (5) under no load condition (the sample is not installed) for various values of magnetic field density magnitude $B_{M}$, where $B_{M}$ is calculated using the following formula:

$$
B_{M}=\frac{E_{S}}{4 W_{S} S f}
$$

where $E_{S}$ is average value of e.m.f. of the measuring winding $[\mathrm{V}] ; W_{S}$ is number of turns of the measuring winding; $S$ is cross-sectional area of the yoke $\left[\mathrm{m}^{2}\right] ; f$ is industrial frequency of $50 \mathrm{~Hz}$.

Hence, under no load condition, the power loss in the magnetic core $P_{S}$ is

$$
P_{S}=P-P_{C}
$$

Under the testing condition, the eddy current loss in the lamination sample produced by the normal flux $P_{E n}$ for various values of $E_{S}$ are calculated by:

$$
P_{\text {En }}=P-\left(P_{C}+P_{S}\right)
$$

At the initial stage, the lamination samples were tested by both experimental setups to obtain the reference values of the power loss $P_{E n}$. Setup 1 was used to test the samples under the opposite flux direction arrangement, whereas setup 2 was employed to conduct the tests under the same flux direction arrangement.

\section{Influence of LengthWise Cuts IN THE LAMINATION SAMPLES}

After the initial investigation of the lamination samples, the ends of the other samples having the same dimensions $(165 \times 100 \mathrm{~mm})$ were cut by a laser cutter in a lengthwise fashion (width of the cuts $\leq 0.2 \mathrm{~mm}$ ) into equal sections (2, 4,8 , and 12 sections). The central part of the samples was not cut for a practical reason (Fig. 7). Fig. 8 illustrates the difference between an uncut lamination sample and a cut sample. The purpose of this experiment is to investigate how the eddy current loss depends on the width of the lamination. The tests were conducted at magnetic field density $B_{M}=(0.1-0.3) \mathrm{T}$.

It can be seen in Fig. 8 that the total width of the samples is $b$, whereas the width of each section (called equivalent width) is $b_{q}$. The ratio of the total sample width to the width of a cut section $N$ is

$$
N=\frac{b}{b_{q}}
$$

In terms of this test, the ratio $N$ is actually the number of the sections cut in the lamination sample. 


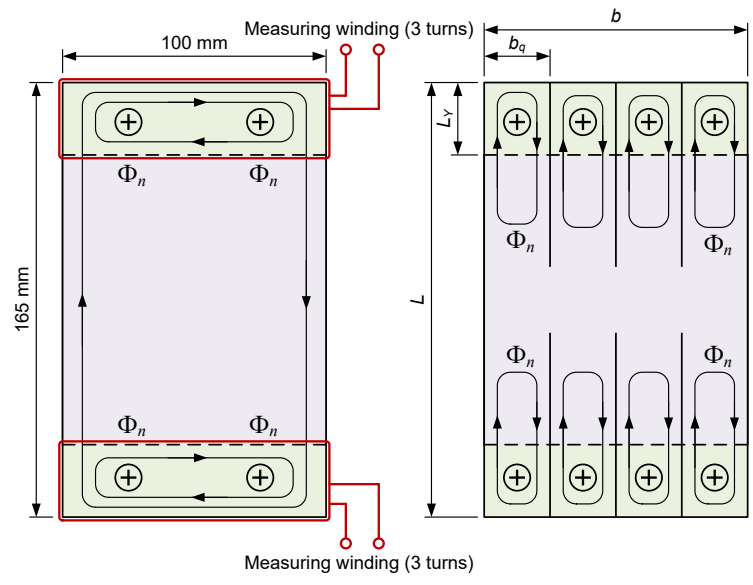

Fig. 8. Uncut and cut lamination samples $(N=4)$.

At the next stage, the cut samples were tested under the same condition that has been applied for the uncut samples. Power loss produced by the normal flux expressed in p.u. for various ratio $N$ can be found as follows:

$$
P_{E n, p u}(N)=\frac{P_{E n}(N)}{P_{E n}}
$$

where $P_{E n, p u}(N)$ is power loss in p.u. produced by the normal flux for a particular ratio $N ; P_{E n}(N)$ is power loss produced by the normal flux for a particular ratio $N[\mathrm{~W}] ; P_{E n}$ is power loss produced by the normal flux for the uncut sample [W].

The test results are summarised in Table I. The curves $P_{E n, p u}$ vs. $N$ for two arrangements of the flux direction (opposite and the same) are shown in Fig. 9a. The curves were approximated using the following expression:

$$
P_{E n, p u}(N)=\left(\frac{1}{N}\right)^{s(\xi)}
$$

where $s(\xi)$ is an exponent depending on the damping factor $\xi$. The value of the exponent $s(\xi)$ can be found from the logarithm expression of (11)

$$
s(\xi)=\frac{\lg P_{E n, p u}(N)}{\lg \left(N^{-1}\right)}
$$

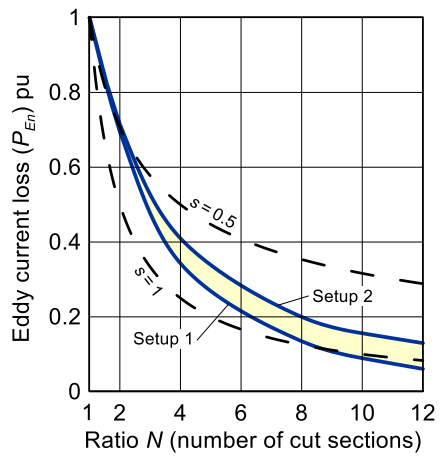

(a)

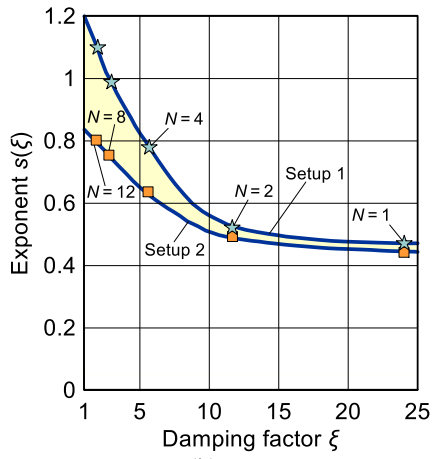

(b)
Fig. 9. Curves (a) $P_{E n, p u}$ vs. ratio $N$ and (b) exponent $s(\xi)$ vs. damping factor $\xi$ for two arrangements of the flux direction (opposite and the same).

table I. Lengthwise Cut Test Results

\begin{tabular}{|c|c|c|c|c|c|c|}
\hline \multicolumn{2}{|c|}{ Ratio $N$} & $\mathbf{1}$ & $\mathbf{2}$ & $\mathbf{4}$ & $\mathbf{8}$ & $\mathbf{1 2}$ \\
\hline \multicolumn{2}{|c|}{ Damping factor $\xi$} & 24 & 12 & 6.0 & 3.0 & 2.0 \\
\hline \multirow{2}{*}{$\begin{array}{c}\text { Setup 1 (opposite flux } \\
\text { direction arraignment) }\end{array}$} & $P_{E n, p u}(N)$ & 1 & 0.695 & 0.343 & 0.135 & 0.061 \\
\cline { 2 - 7 } & $s$ & $\approx 0.48$ & 0.52 & 0.77 & 0.98 & 1.12 \\
\hline $\begin{array}{c}\text { Setup 2 (the same flux } \\
\text { direction arrangement) }\end{array}$ & $P_{E n, p u}(N)$ & 1 & 0.715 & 0.41 & 0.2 & 0.13 \\
\cline { 2 - 7 } & $s$ & $\approx 0.44$ & 0.49 & 0.64 & 0.77 & 0.79 \\
\hline
\end{tabular}

For ratio $N=1$, there is an uncertainty in the form: $0 / 0$; however, it can be overcome using L'Hospital's rule ( $s$ is found as the limit of the ratio of the function increment to the argument increment at $N \rightarrow 1$ ).

The analysis of the curves in Fig. 9a has led to the following conclusions:

- the loss $P_{E n, p u}$ is asymptotically decreased with an increase in $\mathrm{N}$ and following the same pattern for both arrangements of the flux direction (opposite and the same);

- the extent of loss reduction is higher for the opposite arrangement where the experimental setup 1 has a smaller yoke width $L_{Y}$;

- the loss $P_{E n, p u}$ is decreased by more than an order of magnitude at the ratio $N>10 \quad\left(b_{q} \leq 10 \mathrm{~mm}\right)$ corresponding to the damping factor $\xi<4$.

Data in Table I show that the damping factor $\xi$ is decreased with an increase in the ratio $N$. The damping factor reduction is calculated using (2):

$$
\xi(N)=\frac{\xi}{N}
$$

Fig. $9 \mathrm{~b}$ shows the curves $s(\xi)$ vs. $\xi$ for two arrangements of the flux direction (opposite and the same). The curves are actually boundaries of the area of variation of $s(\xi)$. It can be seen that $s \leq 0.5$ is corresponding to the severe skin effect conditions $(\xi>>4)$ whereas $s \geq(0.8-1.0)$ is associated with the reduced skin effect $(\xi<4)$.

Fig. 9a demonstrates two dashed curves obtained using the analytical expressions (11) and (12). These curves illustrate approximate boundaries of the area where the asymptotically decreasing power losses $P_{E n, p u}$ vs. the ratio $N$ are located. The upper boundary is the analytical curve (11) obtained for the fixed exponent $s=0.5$, the lower boundary is a similar analytical curve for $s=1$. It can be seen that the upper curve is the approximate boundary at the ratio $N=(1-2)$ whereas the lower curve is the boundary at the ratio $N=(8-12)$.

Therefore, the influence of lengthwise cuts in the laminations on the power loss in the joint area of a magnetic core can be expressed using the following proportional relationship:

$$
P_{E n} \propto \Phi_{M n}^{2}\left(\frac{b}{N}\right)^{s}
$$

where $\Phi_{M n}$ is magnitude of the normal ac flux; $s=s(\xi)$.

\section{INFLUENCE OF LAMINATION OVERLAP}

At the last stage, the influence of the lamination overlap length in the joints $t_{j}$ was investigated using the experimental setup 2. The sample applied for the overlap tests was a rectangular steel sheet (grade 3406) having the size of $165 \times 100 \mathrm{~mm}$. The overlap area of the test sample depends on installing arrangement of the sample ends into the gaps between the yokes (Fig. 5). The tests were conducted for the overlap length of $26,18,12$, and $6 \mathrm{~mm}$ symmetrically arranged at each end of the sample. The magnetising windings of the setup were connected to an ac voltage source via variac to adjust the fluxes in the cores. The measuring windings were glued at the ends of the sample following the perimeter of the core cross-sectional areas (Fig. 8) to monitor the fluxes through the sample ends. Table II demonstrates the results of experiment: the power losses $P_{E n, p u}$ obtained for various lamination overlaps. 
TABLE II. Lamination Overlap Test Results

\begin{tabular}{|c|c|c|c|c|}
\hline \multirow{2}{*}{$\boldsymbol{P}_{\text {En(aver })}$} & \multicolumn{4}{|c|}{$\boldsymbol{P}_{\text {En } \boldsymbol{p u}}$ at condition $\boldsymbol{\Phi}_{\boldsymbol{M n}}\left(\boldsymbol{t}_{\boldsymbol{j}}\right)=$ const. } \\
\cline { 2 - 5 } & $t_{j}=26 \mathrm{~mm}$ & $t_{j}=18 \mathrm{~mm}$ & $t_{j}=12 \mathrm{~mm}$ & $t_{j}=6 \mathrm{~mm}$ \\
\hline $0.68 \mathrm{~W}$ & 1.02 p.u. & 0.96 p.u. & 0.98 p.u. & 1.04 p.u. \\
\hline $1,11 \mathrm{~W}$ & 1.03 p.u. & 0.99 p.u. & 0.96 p.u. & 1.01 p.u. \\
\hline $1.64 \mathrm{~W}$ & 1.03 p.u. & 0.98 p.u. & 1.02 p.u. & 0.96 p.u. \\
\hline
\end{tabular}

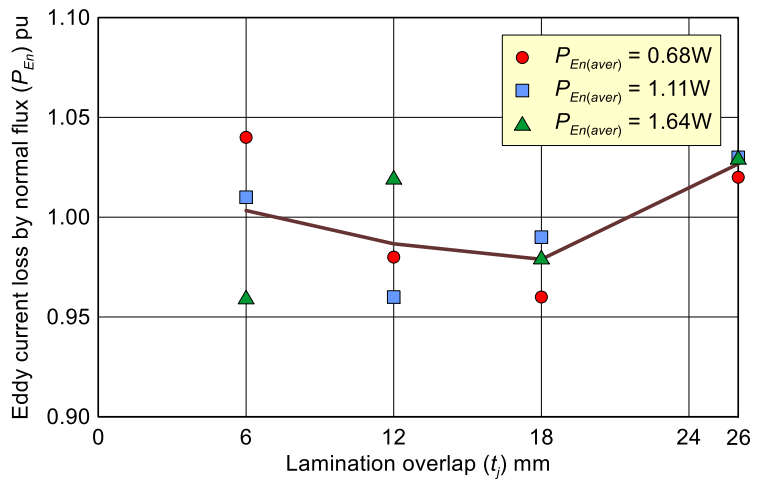

Fig. 10. Power loss in p.u. vs. lamination overlap in $\mathrm{mm}$.

Power loss produced by the normal flux in p.u. is calculates using the formula $P_{E n, p u}=P_{E n} / P_{E n(\text { aver })}$, where $P_{E n(\text { aver })}$ is average value of power loss developed using four readings for the lamination overlaps $t_{j}=6 ; 12 ; 18 ; 26 \mathrm{~mm}$ measured at three different constant fluxes. Fig. 10 shows the power loss in p.u. vs. lamination overlap in $\mathrm{mm}$ for the different constant fluxes. It can be seen that the lamination overlap does not affect significantly on the power loss in the joint area at any value of magnetic flux.

\section{CONCLUSION}

The tests conducted to verify analytical approach have demonstrated that the eddy current power loss produced by the normal flux is independent on the lamination overlap length $t_{j}$. It has been shown that the eddy current loss in the joint area is proportional to the normal magnetic flux $\propto\left(\Phi_{M n}\right)^{2}$ but not to magnetic field density $\left(B_{M}\right)^{2}$ as in (3) and (4). Eddy currents are not limited by the joint area of the laminated core but acting within the entire lamination sheet. The loss is generated under the severe skin effect condition and related to the magnitude of the normal magnetic flux and width of the lamination:

$$
P_{E n} \propto \Phi_{M n}^{2} \times b^{s} \quad \text { where } s \approx 0.5
$$

Hence, the loss produced by the normal flux in "steplap" joints is in four times higher than the power loss the joint area of the similar core assembles using "butt-lap" techniques.

The lengthwise cuts at the ends of the lamination reduce the effective lamination width $b_{q}$ available for the eddy current development. The cuts and reduced skin effect conduction decrease the power loss in the joint area as shown in (16).

$$
P_{E n} \propto \Phi_{M n}^{2} \times b_{q}^{s} \quad \text { where } s \approx 1.0
$$

The results of practical experiments discussed in this paper underpin the analytical approach and provide a better understanding of power loss development in the joint area of the laminated core of power transformers.

\section{REFERENCES}

[1] K.R.M. Nair, Power and Distribution Transformers: Practical Design Guide. Boca Raton, FL: CRC Press, 2021.
[2] A. Moses, P. Anderson, K. Jenkins, and H. Stanbury, Electrical Steels - Volume 2: Performance and Applications. London: Institution of Engineering and Technology, 2019.

[3] S. Tumanski, Handbook of Magnetic Measurement. Boca Raton, FL: CRC Press, 2011.

[4] H. De Keulenaer, D. Chapman, and S. Fassbinder, "The scope for energy saving in the EU through the use of energy-efficient electricity distribution transformers," in Proc. 16th Int. Conf. and Exhibition on Electricity Distribution CIRED-2001, Amsterdam, Netherlands, 1821 June 2001, vol. 4, Art no. 4.27.

[5] V.A. Zaikova, I.E. Startseva, and B.N. Filippov, Domain Structure and Magnetic Properties of Electrotechnical Steels. Moscow: Nauka, 1992.

[6] Y. Kalinin, A. Chivenkov, Y. Vagapov, and A. Anuchin, "Determining specific power loss in joint area of laminated magnetic core," in Proc. 28th Int. Workshop on Electric Drives, Moscow, Russia, 27-29 Jan. 2021, pp. 1-6.

[7] A. Moses, and A. Sakaida, "Effect of distorted flux density on three phase transformer cores assembled from high quality electrical steels," IEEE Trans. on Magnetics, vol. 22, no. 5, pp. 532-534, Sept. 1986.

[8] B.B. Jensen, E.D. Guest, and B.C. Mecrow, "Modeling overlapping laminations in magnetic core materials using 2-D finite-element analysis," IEEE Trans. on Magnetics, vol. 51, no. 6, June 2015, Art no. 7403006.

[9] T. Nakata, N. Takahashi, and Y. Kawase, "Magnetic performance of step-lap joints in distribution transformer cores," IEEE Trans. on Magnetics, vol. 18, no. 6, pp. 1055-1057, Nov. 1982.

[10] T. Nakata, Y. Kawase, and S. Moriyasa, "Magnetic characteristics in the joints of stator cores," IEEJ Trans. on Industry Applications, vol. 107, no. 1, pp. 109-114, 1987.

[11] M. Elleuch, and M. Poloujadoff, "New transformer model including joint air gaps and lamination anisotropy," IEEE Trans. on Magnetics, vol. 34, no. 5, pp. 3701-3711, Sept. 1998.

[12] I. Hernander, F. de Leon, J.M. Canedo, and J.C. Olivares-Galvan, "Modeling transformer core joints using Gaussian models for the magnetic flux density and permeability," IET Electric Power Applications, vol. 4, no. 9, pp. 761-771, 2010.

[13] J. Gyselinck, and J. Melkebeek, "Two-dimensional finite element modelling of overlap joints in transformer cores," COMPEL - The International Journal for Computation and Mathematics in Electrical and Electronic Engineering, vol. 20, no. 1, pp. 253-268, 2001.

[14] Y. Kawase, T. Yamaguchi, and Y. Onogi, "Eddy current analysis of three-phase transformer using 3-D parallel finite element method," in Proc. XXII Int. Conf. on Electrical Machines (ICEM), Lausanne, Switzerland, 4-7 Sept. 2016, pp. 2828-2832.

[15] I. Jamaguchi, Y. Kawase, and S. Ishimura, "Influence of magnetic property of electrical steel sheets of transformer on eddy current loss," Journal of the Japan Society of Applied Electromagnetics and Mechanics, vol. 27, no. 1, pp.67-72, 2019.

[16] G. Bertotti, "General properties of power losses in soft ferromagnetic materials," IEEE Trans. on Magnetics, vol. 24, no. 1, pp. 621-630, Jan. 1988

[17] J. Lammeraner, and M. Stafl, Eddy Currents, G.A. Toombs Ed. London: Iliffe Books, 1966.

[18] M.I. Levin, I.V. Pentegov, S.V. Rymar, and A.V. Lavreniuk, "Analysis of three-phase power transformer laminated magnetic core designs," Electrical Engineering and Electromechanics, no. 1, pp. 4043, 2014.

[19] T. Yagisawa, Y. Takekoshi, and S. Wada, "Magnetic properties of laminated steel sheets for normal fluxes," Journal of Magnetism and Magnetic Materials, vol. 26, nos. 1-3, pp. 340-342, March 1982.

[20] T. Booth, and H. Pfutzner, "Characteristics of transformer core materials for flux normal to the sheet plane," Journal of Magnetism and Magnetic Materials, vol. 133, nos. 1-3, pp. 183-186, May 1994.

[21] N. Hihat, K. Komeza, E. N. Juszczak, and J. P. Lecointe, "Experimental and numerical characterization of magnetically anisotropic laminations in the direction normal to their surface," IEEE Trans. on Magnetics, vol. 47, no. 11, pp. 4517-4522, Nov. 2011.

[22] W. Wang, A. Nysveen, and N. Magnusson, "Eddy current loss in grain-oriented steel laminations due to normal leakage flux," IEEE Trans. on Magnetics, vol. 57, no. 6, June 2021, Art no. 6301604. 